\title{
Willi Semmler \\ Zu neueren Tendenzen in der Theorie und Praxis \\ der amerikanischen Wirtschaftspolitik ${ }^{1}$
}

\section{(I) Einleitung}

Die wirtschaftspolitische Diskussion in den USA seit Mitte der 70er Jahre ist zunehmend von einem Thema bestimmt: Die abfallende Wachstumsrate der Arbeitsproduktivität der US-Industrien und die schwindende internationale Konkurrenzfähigkeit amerikanischer Firmen im Vergleich mit westeuropäischen und japanischen Unternehmen. Dieses Problem war das Hauptproblem, dem sich die Carter-Regierung gegenübersah. Dieses Problem wird auch das Hauptproblem der Reagan-Regierung sein.

Zahlreiche Studien sind angefertigt worden, die die Ursache und das Ausmaß dieses Niedergangs in der Produktivitätsentwicklung zu erfassen und zu erklären suchen. Das weitgehend übereinstimmende Urteil ist: »While the United States experienced the highest growth rate among the industrial nations during 1870-1950, its relative performance was below average for 1950-65 period, and it had the lowest rate during 1965-71.«(Kamrany / Chereb 1979, 166) Der Abfall der Wachstumsrate der Arbeitsproduktivität setzte sich auch in den 70er Jahren fort: Die Wachstumsrate der Arbeitsproduktiviät fiel von 1973-76 auf $0,5 \%$ (vgl. Denison 1979, 2), um dann wieder leicht anzusteigen. In der Nachkriegszeit noch wies der amerikanische Kapitalismus das doppelte Produktivitätsniveau europäischer Länder auf. Frankreich z.B. hatte 1950 ein Bruttosozialprodukt pro Kopf der Bevölkerung, das nur $58 \%$ desjenigen der USA betrug, und die BRD erreichte 1950 nur $50 \%$ des Bruttosozialprodukts pro Kopf der Bevölkerung, gemessen am USA-Niveau. (Statistical Abstracts 1979, 894). Dieser Vorsprung der US-Wirtschaft vor der europäischen schwindet mehr und mehr in den 60er und 70er Jahren. Ende der 70er Jahre ist der Produktivitätsvorsprung der US-Wirtschaft vor der europäischen speziell vor der westdeutschen - aber auch der japanischen - drastisch zusammengeschmolzen. Die meisten empirischen Studien, die über die US-Ökonomie angefertigt wurden, weisen darauf hin, daß im wesentlichen die abfallende interne Kapitalbildung - begleitet von einer abfallenden Wachstumsrate des technischen Fortschritts - den Abfall in der internationalen Konkurrenzfähigkeit verursacht habe (vgl. Denison 1979, Kamrany / Chereb 1979, Feldstein 1973 und die jüngeren Veröffentlichungen des Brookings-Instituts) ${ }^{2}$.

Während die BRD-Wirtschaft in den 50er und 60er Jahren noch durchschnittliche Wachstumsraten der Kapitalbildung von 6-9 \% aufweist (vgl. Altvater / Hoffmann / Semmler 1980, 96), liegen die durchschnittlichen Wachstumsraten des Kapitalstocks in den USA immer unter $5 \%$. Sie gehen Ende der 50 er bis Mitte der 60er Jahre sogar auf ca. 3-4 \% zurück, um dann schließlich in den 70er Jahren auf 3,5\% und von $1975-78$ auf $2,9 \%$ zu schrumpfen. Auch die Wachstumsrate der Kapitalintensivierung der Produktion liegt bei weitem unter der der BRD. In den USA liegt sie in den 50er und 60er Jahren um 2,5-3\%, um dann in den 70er Jahren auf $2 \%$ und dann schließlich auf $1 \%$ abzufallen, während die BRD Raten der Kapitalintensivierung aufweist, die stets über $5 \%$ liegen und die in 
den 70er Jahren noch ansteigen (vgl. Altvater / Hoffmann / Semmler 1980, 91). Mit dem Abfall der internen Kapitalakkumulation in den USA nehmen andererseits aber die Investitionen der großen amerikanischen Konzerne im Ausland rapide zu. 1951 hatten die amerikanischen Konzerne einen Bestand an Auslandsinvestitionen von 6 Mrd. Dollar, 1973 waren es schon $73 \mathrm{Mrd}$. Dollar. Der Bestand an ausländischen Direktinvestitionen als Anteil am BSP stieg von $4.5 \%$ (1951) auf 7,5\% im Jahre 1974 (Seltzer 1978, 38). Demgegenüber war der Anteil der ausländischen Direktinvestitionen am BSP für die BRD in den 50er Jahren nahezu verschwindend gering und betrug auch 1973 nur 3,5\%. (Jonas 1976, Statistisches Jahrbuch 1976).

Zwar wurde dieser Prozeß der abfallenden internationalen Konkurrenzfähigkeit der inländisch operierenden amerikanischen Konzerne in den 70er Jahten noch durch drastische Dollarabwertungen aufgehalten, trotzdem aber hat dieser Prozeß bereits in den 70er Jahren folgende Resultate gezeigt: ${ }^{3}$

- Die europäischen Konkurrenzländer haben ihren Produktivitätsrückstand, den sie noch nach dem Zweiten Weltkrieg aufwiesen, weitgehend aufgeholt. 1977 weist z.B. Frankreich verglichen am USA-Niveau bereits $88 \%$ des BSP pro Kopf der Bevölkerung und die BRD bereits $95 \%$ auf (Statistical Abstracts 1979, 894). Seitdem dürfte der Vorsprung der USA noch weiter zusammengeschmolzen sein, und viele Industriezweige wiesen bereits in den 70er Jahren eine höhere Arbeitsproduktivität auf als diejenigen der USA (vgl. Mayer 1973).

- Der Anteil der USA am gesamten Weltexport schrumpft von (1960) $15,9 \%$ auf (1977) $10,7 \%$, während der Anteil der BRD am gesamten Weltexport von (1950) $3,5 \%$ auf (1977) 10,5\% ansteigt. Diese Verschiebung in den Exportanteilen wird noch deutlicher, wenn nur die Exporte der verarbeitenden Industrie betrachtet werden. Die BRD kann von 1964 bis 1977 ihren Anteil am Weltexport von Industrieprodukten von $19,3 \%$ auf $20,8 \%$ steigern, die USA fällt im gleichen Zeitraum von $21,5 \%$ auf $15,9 \%$ zurück. Die BRD hat die USA nun überflügelt und ist zum führenden Exportland für Industrieprodukte, insbesondere Investitionsgüter, geworden.

- Mit den sinkenden Raten des Produktivitätswachstums - die in den Jahren 1973-1975 negativ wurden - mit dem Anstieg der Arbeitslosentate auf $8 \% 1975$, ca. 6-6,5\% in der zweiten Hälfte der 70er Jahre und ca. 8-8,5\% zu Beginn der 80er Jahre und mit dem Ansteigen der Inflationsraten (die 1974 bereits bei $11 \%$ lagen), wurden die Wachstumsraten des Reallobns der amerikanischen Arbeiter zum ersten Mal in der Nachkriegsgeschichte des amerikanischen Kapitalismus negativ (1973-75 gleich ca. $-0,3 \%$ ). Der durchschnittliche Reallohn der amerikanischen Arbeiter ist 1980 niedriger als vor 15 Jahren, und der Lebensstandard breiter Schichten amerikanischer Arbeiter ist unter den der europäischen Konkurrenzländer gefallen.

- Bedingt durch den schwindenden Kokurrenzvorteil der US-Wirtschaft und den Anstieg der Handels- und Zahlungsbilanzdefizite (die Handelsbilanz ist negativ seit Ende der 60er Jahre, die Zahlungsbilanz war schon früher negativ) findet aber auch eine allmähliche Entthronung des US-Dollars als internationales Zirkulations- und Wertaufbewahrungsmittel statt. 1971 wurde die Goldkonvertibilität des Dollars und 1973 die fixen Wechselkurse aufgehoben. Der Verlust der ökonomischen Vormachtstellung der USA auf dem Weltmarkt drückt sich auch in dem rapiden Verlust der Hegemonialstellung des Dollars aus.

- Ein weiteres gravierendes Problem stellten die seit den 70er Jahren auf zweistellige 
Ziffern angewachsenen Inflationsraten dar. Diese zweistelligen Inflationsraten können jedoch weder aus den Lohnzunahmen (die Löhne stagnierten, und der reale Wochenlohn amerikanischer Arbeiter ist niedriger als vor 15 Jahren), noch aus den staatlichen Defiziten, noch aus der Geldmengenzunahme, noch allein aus den Erdölpreissteigerungen abgeleitet, sondern müssen vielmehr aus den mangelnden Produktivitätszunahmen der amerikanischen Industrie erklärt werden (vgl. Okun / Perry 1978 und Houthacker 1979).

Während dieses Prozesses der Erosion der Konkurrenzfähigkeit der USA seit Beginn der 60er Jahre hatte aber auch gleichzeitig die Internationalisierung der Produktion, die internationale Kapitalmobilität und die (störende) Rolle des internationalen Spekulationskapitals so stark zugenommen, daß die Geld- und Fiskalpolitik - wie sowohl orthodoxe Ökonomen als auch Politökonomen konstatierten - nicht mehr als hinreichende Instrumente zur Regulierung der amerikanischen Wirtschaft erschienen. Die unter Kennedy 1961 eingeführte keynesianische Politik des Demandmanagements - die in den 50er Jahren trotz hoher Arbeitslosenquoten nicht angewandt wurde - war bis Mitte der 70er Jahre Eckstein der amerikanischen Wirtschaftspolitik gewesen. Sie wurde überdies noch 1971 unter Nixon von der Preis- und Einkommenspolitik ergänzt. In diesem ganzen Zeitraum war jedoch die Fiskalpolitik immer wesentliches Instrument der Wirtschaftspolitik. Nur die Geldpolitik durchgeführt im wesentlichen von den Federal Reserve Banks der Einzelstaaten - war bereits nach monetaristischem Muster gestaltet. Mit dieser keynesianisch orientierten Wirtschaftspolitik - die jedoch mehr und mehr als Stabilisierungspolitik gegen das steigende Preisniveau eingesetzt wurde - wurde das Wachstum der Produktion, der Produktivität und die Kapitalakkumulation im Inland und im Ausland als gegeben unterstellt. Gerade aber auf dieser Seite hatten sich - wie oben gezeigt - wesentliche Veränderungen vollzogen, die zu gravierenden Problemen der amerikanischen Wirtschaftspolitik der 70er Jahre wurden. Die oben diskutierten Änderungen der internen Bedingungen der Wirtschaftsentwicklung als auch die Änderungen det externen Bedingungen wie die ansteigende internationale Kapitalmobilität, insbesondere der amerikanischen multinationalen Konzerne, die Mobilität des internationalen Spekulationskapitals, machten den amerikanischen Kapitalismus immer unregulierbarer durch eine Politik des Nachfragemanagements.

Ein einflußreicher Vertreter der amerikanischen Keynesianer beschreibt daher 1980 die Situation der USA mit folgenden Worten:

»... the role of the United States in the world economy has changed significantly. The spread of technology, increased mobility of capital, and industrialization in other countries have sharply reduced the dominating position of the United States in many of its traditional markets, and the growth of financial centers abroad has reduced foreign dependence on the dollar. Today there is a more open competitive world economy with a high degree of dependency among the countries. $« \ldots »$ The sharply expanded role of external factors during the 1970 s... created a dilemma for demand management policies.« (Bosworth 1980, 36, 39)

Angesichts dieser Veränderungen der internen wie der externen Bedingungen der Wirtschaftsentwicklung der U.S.A. erwies sich die keynesianische Stabilisierungspolitik nicht mehr als wirksam, Produktion, Beschäftigung und Preisniveau makroökonomisch zu steuern. Aus diesen Gründen bricht auch bereits in den 70er Jahren in den USA eine scharfe Kontroverse um Notwendigkeit und Effektivität der keynesianischen Stabilisierungspolitik aus, auf die ich zuerst eingehen möchte. 


\section{(II) Zu den theoretischen Kontroversen um die Stabilisierungspolitik}

Die mehr orthodoxe amerikanische Diskussion zur Theorie der Wirtschaftspolitik wird auf zwei Ebenen geführt (auf die Einschätzung der Wirtschaftspolitik durch die amerikanischen Gewerkschaften gehe ich im letzten Teil ein):

- $\quad$ zum einen geht es in der Diskussion um die Frage der mikroökonomischen Basis der Makroökonomie;

- $\quad$ zum anderen geht es in der Kontroverse um die Notwendigkeit und Effektivität der makroökonomischen Stabilisierungspolitik selbst.

Sowohl auf der ersten als auch auf der zweiten Ebene liefern sich neoklassische / monetaristische Vertreter der ökonomischen Theorie mit Keynesianern und Neo-Keynesianern heftige Gefechte.

\section{(II) 1. Zur mikroökonomischen Basis der Makroökonomie}

Die erste Ebene der Diskussion um die Stabilität oder Instabilität der kapitalistischen Marktwirtschaften bezieht sich auf die Frage der adäquaten mikroökonomischen Basis der Makroökonomie. Die Keynesische Theorie und die traditionelle Makroökonomie der Lehrbücher der Nachkriegszeit hatten die Frage des mikroökonomischen Verhaltens von Unternehmern und Konsumenten bei makroökonomischer Stabilisierungspolitik des Staates überwiegend ausgeklammert. Nicht nur wurde die Produktions- und Angebotsseite bei der Keynesischen Betrachtung als gegeben unterstellt, sondern es wurde auch nicht danach gefragt, ob das Verhalten von Einzelunternehmen oder hochkonzentrierten, international operierenden Konzernen bei makroökonomischer Steuerung Reaktionen zeigt, die die makroökonomischen Ziele erfüllen helfen. Tobin, ein bekannter amerikanischer Keynesianer, beschreibt diesen Gesichtspunkt der Kritik folgendermaßen: „One view is ... that traditional macroeconomic theory suffers from its lack of firm microeconimic foundations. The behavioral relations of macro models ... are not rigorously derived from optimization by individual agents ...«(Tobin 1978, 1). Auf der Diskussionsebene der mikroökonomischen Fundierung der Makroökonomie finden wir gegenwärtig drei Positionen.

1. Neoklassische, an Walras orientierte allgemeine Gleichgewichtstheoretiker behaupten, daß Preise auf allen Märkten flexibel sind und das Marktsystem, wenn es ungestört wirken kann, Gleichgewichte von Angebot und Nachfrage auf allen Märkten erzeugt. Zugestanden wird aber, daß auf dem Arbeitsmarkt »Marktfehler« vorliegen. Diese Marktfehler auf dem Markt für die Ware Arbeitskraft bewirken, daß die Preise, d.h. hier die Löhne, nicht flexibel genug sind oder zu lange Anpassungszeiten an Gleichgewichtslöhne aufweisen, so daß es für längere Zeit zu einem Überschuß an Arbeitskräfteangebot, d.h. zur Arbeitslosigkeit kommen kann (vgl. Solow 1979). Faktoren, die Marktfehler auf seiten der Ware Arbeitskraft in den Augen dieser Theoretiker begründen, sind:

- die Monopolmacht der Gewerkschaften (d.h. die gewerkschaftliche Organisierung der Arbeiter, vgl. schon Pigou, Hicks in den 30er Jahren);

- das Suchverhalten von Arbeitern auf dem Arbeitsmarkt (Phelps 1970);

- die langen Kontrakte zwischen Arbeitern (oder Gewerkschaftern) und Unternehmen (Kontrakttheorie des Arbeitsmarktes, Fisher 1977, Baily 1978, Pool 1976).

Infolge dieser Faktoren sind gemäß den neoklassischen Gleichgewichtstheoretikern die 
Löhne rigide und passen sich nicht den Gleichgewichtslöhnen an, bei denen Angebot und Nachfrage nach Arbeit ausgeglichen wären. Ein keynesianisches Gleichgewicht bei Unterbeschäftigung kann die Folge sein.

2. Diese von den Keynesianern kaum ernstgenommenen neoklassischen Gleichgèwichtstheoretiker finden jedoch Verstärkung durch eine neue Gruppe von Walrasianern, die die keynesianischen Ungleichgewichts- und Instabilitätsanalysen ernstnehmen, diese aber auf eine mikroökonomische (walrasianische) Basis stellen wollen. Diese keynesianisch orientierten Walrasianer lassen die Fiktion des Tausches - des Kaufs und Verkaufs von Waren - zu Gleichgewichten fallen und analysieren den Tausch, wenn Ungleichgewichtspreise vorliegen. Ihr Konzept ist, daß der Kauf und Verkauf der Waren - und auch der Ware Arbeitskraft - zu Preisen stattfindet, die nicht Gleichgewichtspreise sind. Alle Preise und auch die Löhne können für Tauschprozesse als gegeben und kurzfristig als rigide angenommen werden. Nur noch Mengenreaktionen bestimmen das Verhalten der Marktagenten - d.h. z.B. das Verhalten von profitmaximierenden Unternehmen und nutzenmaximierenden Verkäufern von Arbeitskraft. Wie eine Reihe von Theoretikern gezeigt hat (vgl. Barro / Grossmann 1971, Mainvaud 1977, Grandmont / Laroque 1977, Clower 1965, Leijonhufvud 1968, Benassy 1975, Fisher 1977) kann bei kurzfristig gegebenen und rigiden Preisen und Löhnen

- ein Gleichgewicht auf den Gütermärkten (bei rigiden Preisen produzieren die Unternehmen nur bestimmte Mengen, auch wenn die Kapazitäten nicht ausgelastet sind); - ein Gleichgewicht von Sparen und Investieren (die Anpassung von Sparen und Investieren verläuft entsprechend der Keynesischen Vorstellung über die Einkommensveränderung nicht über den Zinssatz), und

- ein Ungleichgewicht auf dem Arbeitsmarkt, d.h. unfreiwillige Arbeitslosigkeit zustande kommen.

Von diesen Theoretikern wird ein Keynes-Gleichgewicht - das ein Ungleichgewicht auf dem Arbeitsmarkt einschließt - konsequent auf dem Boden der neoklassischen allgemeinen Gleichgewichtstheorie abgeleitet. Als vorherrschender Mechanismus wird jedoch ein Mengenanpassungs- und nicht ein Preisanpassungsmechanismus angenommen. Wenn der Ansatz auch problematisch ist für Keynesianer, da er profit- und nutzenmaximierendes Verhalten von Firmen bzw. Konsumenten unterstellt - was ja die Keynesianer ablehnen -, so scheint ein wesentlicher Punkt - der auch durch vielfache empirische Untersuchungen erhärtet wurde (vgl. Semmler 1979) - eine realistische Annahme zu sein: nämlich die Annahme, daß die Preise kurzfristig rigide sind.

3. Gerade diesen Punkt macht eine dritte Gruppe von Theoretikern zum wesentlichen Punkt ihrer mikroökonomischen Begründung der Makroökonomie. Diese dritte Gruppe von Theoretikern, die als Post-Keynesianer bezeichnet werden können, geht von der weitgehenden Oligopolisierung in der verarbeitenden Industrie aus (vgl. Sylos-Labini 1969, Weintraub 1979, Eichner 1976, Harcourt 1977). Aufgrund dieser Annahme, die sie auch preistheoretisch zu begründen versuchen, können die Keynesschen Multiplikator- und Akzeleratorprozesse als Mengenprozesse analysiert werden, die wesentlich die Instabilität des Kapitalismus begründen. Wenn Oligopolpreise auf den Märkten vorherrschen, setzen sich Schwankungen in der Investitionstätigkeit der Unternehmen - diese Schwankungen in der Investitionstätigkeit waren für Keynes der zentrale Punkt der Konjunkturanalyse - in Schwankungen von Produktion, Beschäftigung und Einkommen um, ohne daß Preisbewegungen Gegenbewegungen darstellen können. Das empirische Faktum der kurzfristigen 
Rigidität von Preisen scheint auch hier in dieser Theorie eine realistische Annahme zu sein. Problematisch ist allerdings, ob die Rigidität von Preisen durch den Oligopolansatz begründet werden muß (vgl. Semmler 1979).

Ist diese Diskussion um die mikroökonomische Begründung der Makroökonomie zwar nicht unmittelbar relevant für wirtschaftspolitische Maßnahmen, so wird von hier aus jedoch die Notwendigkeit der Stabilisierungspolitik begründet (Keynesianer) oder bestritten (Neoklassiker / Monetaristen).

\section{(II) 2. Notwendigkeit und Effektivität der Stabilisierungspolitik}

Wirtschaftspolitisch viel relevanter als die Diskussion um die mikroökonomische Begründung der Makroökonomie ist allerdings in den 70er Jahren die Diskussion um die Notwendigkeit und Effektivität der Stabilisierungspolitik gewesen. Aufgrund der oben dargestellten Erfahrungen bei der Anwendung des Keynesianismus seit der Kennedy - Ära ist die Stabilisierungspolitik von konservativer Seite mehr und mehr unter Beschuß gekommen. Auch auf dieser Ebene der Diskussion waren bekanntermaßen die Neoklassiker / Monetaristen die Hauptopponenten der Keynesianer.

\section{Monetaristen und Rational-Expectation-Schule}

Wie bekannt ging ja bereits der Monetarismus davon aus (Friedmann 1968), daß

- das ökonomische System bei flexiblen Preisen zur Selbstregulierung neigt;

- die Geldpolitik einen stärkeren Einfluß auf die Wirtschaftsentwicklung hat als die Fiskalpolitik;

- eine unstetige Geldpolitik und Fiskalpolitik (z.B. in den 60er und 70er Jahren in den USA) eine unstetige Wirtschaftsentwicklung erst erzeugt hat;

- nur ein kurzfristiger trade-off zwischen Arbeitslosigkeit und Inflation besteht (Phillips-Kurve), d.h. bei adaptiver Anpassung an die richtige Inflationsrate würde langfristig kein trade-off bestehen und die natürliche Rate der Arbeitslosigkeit kann nicht beseitigt werden;

- cine staatliche Nachfragepolitik (finanziert etwa durch dic Ausgabe von Wertpapieren oder Geldschöpfung) entweder nur die Inflation anheizen oder die privaten Investitionen verdrängen wird (crowding-out), und

- $\quad$ nur eine Politik der konstanten Geldmenge den Wirtschaftsprozeß stabilisieren kann (Friedman 1968).

Zahlreiche theoretische und ökonometrische Studien, die von monetaristischer Seite vorgenommen wurden, sollten vor allem die Notwendigkeit der staatlichen Stabilisierungspolitik zurückweisen helfen (vgl. Modigliani 1977). Daß die Stabilisierungspolitik wirksam sein könnte, wurde weitgehend nicht bestritten, nur erzeuge sie die falschen Wirkungen (Erzeugung von Instabilitäten). Neuerdings wird aber die Stabilisierungspolitik nicht nur von Moneratisten, sondern auch von einer ultrakonservativen Richtung der ökonomischen Theorie heftig angegriffen. (Barro 1974, Lucas 1973, Sargent/Wallace 1976, Sargent 1976, Muth 1961). Diese neue Schule, die sich Rational-Expectation-Schule oder »New Classical Macro-Economics« nennt, bestreitet auch die Effektivität der Geld- und Fiskalpolitik, die natürliche Rate der Arbeitslosigkeit zu beeinflussen. Diese Richtung, die auch den Monetarismus noch kritisiert, weil er die Effektivität der Stabilisierungspolitik akzeptiere, ver- 
sucht mit umfangreichen ökonometrischen Tests vor allem die beiden folgenden Thesen zu belegen:

- Erstens: Die Geldpolitik hat keinen Effekt auf die Arbeitslosenrate, wenn die Marktteilnehmer unter der Bedingung tationaler Erwartungen handeln. Wenn das Ansteigen der Geldmenge durch Geldmengenpolitik Preissteigerungen verursacht, die von den Gewerkschaften oder Arbeitern in ihren Lohnforderungen voll antizipiert werden, bleiben zusätzliche Gewinne für die Unternehmen aus, und diese Politik hat keinen Effekt auf die Rate der Arbeitslosigkeit.

- Zweitens wird behauptet, daß auch die Fiskalpolitik keinen Effekt auf die Gesamtnachfrage und damit das Beschäftigungsniveau hat. Wenn die zusätzliche staatliche Nachfrage bei Defizit-Spending über die Ausgabe von Staatspapieren finanziert wird, (vgl. Mundel 1971, 13), dieser Anstieg der Staatsschuld aber bei den Marktteilnehmern den Effekt hat, daß sie zukünftige Steuererhöhungen antizipieren, bleiben auch die Effekte auf die Gesamtnachfrage aus, und die natürliche Rate der Arbeitslosigkeit kann nicht verändert werden. Ähnliche Effekte hat auch die Finanzierung der zusätzlichen staatlichen Nachfrage durch Geldschöpfung.

Während also die Monetaristen nur die Notwendigkeit der Stabilisierungspolitik bestritten, nicht aber die Effektivität, bestreiten die neuen stark konservativ orientierten Vertreter der Rationalen-Erwartungs-Hypothese die Notwendigkeit und die Effektivität der Wirtschaftspolitik.

\section{Die Antwort der Keynesianer}

Herausgefordert durch die konservative und ultrakonservative Kritik mußten die Keynesianer zum Gegenangriff übergehen. Ihte zentralen Thesen hierbei waren (vgl. Blinder / Solow 1973 und 1974, Tobin 1978, Modigliani 1977, B. Friedmann 1978):

- Eine ausschließliche Geldpolitik - und etst recht eine Politik der konstanten Geldmenge - kann nicht die inhärenten Instabilitäten des Marktsystems oder von außen kommende Strömungen (»supply shocks«) des Marktsystems effek tiv beseitigen (Modigliani 1977).

- Die Fiskalpolitik ist wesentliches Instrument der Stabilisierungspolitik und unter der Bedingung unterausgelasteter Kapazitäten ist dic Verdrängung privater Aktivitäten (Investitionen) durch Fiskalpolitik auch nur minimal (Blinder / Solow 1973, B. Friedmann 1978).

- Die Annahme der rationalen Erwartungsbildung bei den Marktteilnehmern, also die Annahme, daß Marktteilnehmer langfristige Trends von Preisen und Mengen durch stochastische Störungen hindurch mittels ständiger ökonometrischer Tests erfassen können, ist nicht haltbar. Sowohl die Annahme der ständigen Prognose markträumender Gleichgewichtspreise durch die Marktagenten als auch die Hypothese, daß die Schwankungen in der natürlichen Rate der Arbeitslosigkeit nur von den Schwankungen des Arbeitsangebots herrühre, die von der Wirtschaftspolitik nicht beeinflußt werden kann, sind für Keynesianische Denkweisen unrealistische Hypothesen (Tobin 1978).

Selbst nach dieser neuesten Diskussion um die Notwendigkeit und Effektivität der Wirtschaftspolitik, bei der von den Kritikern mehr und mehr nach der soliden mikroökonomischen Basis der Makroökonomic gefragt wurde, hatten die Keynesianer aber keineswegs das Feld geräumt. In der Wirtschaftspolitik der USA in den 70er Jahren z.B. durch die Gutach- 
tertätigkeit für das 》oint Committee im Kongreß, im Council of Economic Advisors, in einflußreichen Wirtschaftsforschungsinstituten (Brookings Institution, National Bureau of Economic Research) hatten die keynesianisch orientierten Theoretiker noch einen stärkeren Einfluß bewahren können als die Monetaristen. Die Monetaristen selbst haben nur einen gewissen Einfluß in den Zentralbanken der Einzelstaaten und im Federal Reserve Board. Trotz dieser erneuten erfolgreichen Verteidigung der Stabilisierungspolitik durch die Keynesianer hatten sich aber auch einflußreiche Keynesianer dem monetaristischen Programm angenähert. In den Reihen der Keynesianer, insbesondere bei einer starken konservativen Fraktion, war eine zunehmende Skepsis entstanden, ob die Stabilisierungspolitik unter den sich verändernden Bedingungen für die US-Wirtschaftspolitik - wie die starke internationale Verflechtung der US-Wirtschaft, die zunehmende internationale Kapitalmobilität und die abnehmende internationale Konkurrenzfähigkeit - überhaupt noch sinnvoll und wirksam sei oder vielmehr die Politik der Nachfragesteuerung durch eine Politik der Produktions- und Angebotsförderung ergänzt oder ersetzt werden müsse.

Diese aus der keynesianischen Richtung selbst hervorgegangenen mehr Angebots orientierten Ökonomen waren in den 70er Jahren unter der Carter-Regierung sehr einflußreich gewesen, nicht nur unter liberalen Kongreßmitgliedern und dem Joint Committee des Kongresses, sondern auch in den Gewerkschaften. Ihr Hauptaugenmerk gilt mehr und mehr wie oben erwähnt - vor allem der Weltmarktposition der USA. Die Thesen dieser konservativen Keynesianer lassen sich in fünf Punkten zusammenfassen:

- Sie halten im Gegensatz zu den Monetaristen und den Vertretern der Rationalen-Erwartungs-Hypothesen an der Funktion der Stabilisierungspolitik, insbesondere der Fiskalpolitik, durchaus fest. Sie sehen aber die Bedingungen gewandelt, unter denen diese Politik effektiv sein könnte. Angesichts der sinkenden Konkurrenzfähigkeit der USA, der niedrigen Wachstumsraten der Produktivität und der Kapitalbildung, der internationalen Interdependenzen, dem permanenten Zahlungsbilanzdefizit und dem verfallenden Dollar und dem gleichzeitigen Anstieg von Arbeitslosigkeit und Inflation erreicht die keynesianische Politik der Nachfragesteuerung ihre Grenzen. Für sie wird die Förderung der Produktions- und Angebotsseite nun relevanter: »We must shift our attention to the supply side of our economy and, in the determination of President John Kennedy, 'get our country moving again' . (Chairman of the Joint Committee, 1979).

- Mit den konservativen Monetaristen teilen sie die Auffassung, daß seit den 60er Jahren die Ökonomie übereguliert, überstimuliert und die Marktagenten, insbesondere die Unternehmen, mit Regulierungsauflagen zu stark belästigt wurden und die hohen Steuern die in den 70er Jahren notwendige Kapitalbildung verhindert haben. Die finanziellen Kosten der staatlichen Intervention insbesondere im Bereich des Umweltschutzes, der sozialen Sicherung, der Arbeitssicherheit, des Gesundheitswesens und des Transport- und Kommunikationswesens (vgl. Economic Report of the President, 1980, 119) haben die Kapitalbildung zu stark belastet und Produktivitätssteigerungen verhindert. Industrien müßten wieder dereguliert (Fluggesellschaften, Transportindustrie, Bahn) und die staatlichen Interventionen wieder reduziert werden.

- Die konservativen Keynesianer gehen davon aus, daß der Einsatz der Stabilisierungspolitik zur Senkung der Inflationsrate (etwa über das Einleiten einer Krise) unter den gegenwärtigen Bedingungen ein zu riskantes Mittel für die Wirtschaftspolitik sei. Es wird argumentiert, daß nur eine scharfe und lang anhaltende Krise die Inflationsrate 
und die Löhne herabdrücken würde, die Kosten einer solchen Krise aber zu hoch seien, da gleichzeitig dabei die Investitionen und die Kapitalbildung sowie langfristig die Arbeitsproduktivität zurückgehen und dies die amerikanische Position auf dem Weltmarkt noch mehr verschlechtern würde.

- In den Augen dieser mehr Angebots orientierten Theoretiker ist daher nicht eine erneute Rezession ein Mittel der Bereinigung der Probleme der amerikanischen Wirtschaft, sondern nur die forcierte Kapitalbildung und die Steigerung der Wachstumsrate der Arbeitsproduktivität. Um dies zu erreichen, solite das Steuersystem umstrukturiert und die Kapitalbildung mit billigen Krediten gefördert werden (progressive Abschreibung, Wegfall der Doppelbesteuerung von Aktienbesitzern, Anreize zur erhöhten Spartätigkeit; vgl. Bosworth 1980, Feldstein 1973). Eine Phase der rapiden Kapitalbildung würde nicht nur die Produktivität wieder anheben und die Weltmarktposition verbessern, sondern auch die Inflationstate drücken. (In der Tat sind von diesen Ökonomen bereits empirische Studien vorgelegt worden, die einen negativen Zusammenhang von Wachstumsrate der Produktivität und Inflationsrate konstatieren; vgl. Houthakker 1979).

- In einer solchen Phase der rapiden Kapitalbildung und des Wachstums müßten natürlich Lohn-Preis-Kontrollen eingeführt werden, um den Preis- und Lohnanstieg zu stoppen. Da die andere Alternative - die Rezession als Mittel der Bereinigung - abgelehnt wird, ist dies nur konsequent. "There ist no policy that can break the current momentum of wage-price spiral without high costs, yet a continuation of recent trends is also costly. A mild recession is too weak and mild income programs have lost their credibility... Given a desire to slow the inflation the choice is rapidly being reduced to one of servere recession versus wage-price controls.« (Bosworth 1980, 66)

Die Position dieser mehr Angebots orientieren Theoretiker, die ich als konservativen Keynesianismus bezeichnen möchte, hatte Anklang gefunden bei den ökonomischen Beratern des Präsidenten und bei Kongreßmitgliedern, war aber auch teilweise in die in jüngster Zeit praktizierte Wirtschaftspolitik der Carter-Administration eingegangen (vgl. Teil III).

\section{Supply-Side-Ökonomen}

Wie oben gezeigt, haben sich die theoretischen Konzeptionen zur Wirtschaftspolitik in den 70er Jahren mehr und mehr von Nachfrage orientierten auf Angebots orientierte Konzeptionen verschoben. Diese Verschiebung in der Orientierung der Wirtschaftspolitik ist besonders radikal von neokonservativen neoklassischen Ökonomen - repräsentiert etwa durch das Hoover Institut der Stanford Universität in Kalifornien und das American Enterprise Institut in Washington - in den 60er und 70er Jahren vorangetrieben worden. Diese neue gegenwärtig sehr starke Richtung in den theoretischen Diskussionen zur Wirtschaftspolitik hatte bereits in den 60er und 70er Jahren die Keynesianer in zahlreichen Aufsätzen und Büchern angegriffen. Diese neue Richtung - deren Vertreter sich Supply-Side-Ö́konomen nennen - ist aber dadurch sehr einflußreich geworden, daß sie im wesentlichen das ökonomische Wahlprogramm von Reagan formulierte und auch die zukünftige praktische Wirtschaftspolitik der Reagan-Administration - insbesondere die Geld-Budget- und Steuerpolitik - maßgeblich bestimmen wird. Vertreter dieser Richtung sind etwa Ture 1976, Laffer 1979, Roberts 1978, Mundel 1971, Wanniski 1978. Populäre Versionen des Programms der Supply-Side-Ökonomen werden insbesondere von Laffer 1979 verbreitet. Ihr radikal neokonservatives Programm läßt sich in drei Punkten zusammenfassen: 
- Entsprechend ihrer Auffassungen hat der Keynesianisch orientierte Steuer- und Ausgabenstaat zu einem Niedergang der produktiven Aktivitäten geführt »Macroeconomic Policy in much of the W/estern world today consists of taxes on work, output, and employment in conjunction with subsidies to nonwork, leisure and unemployment. Thus should come as no surprise that much of the Western world today has little work output and employment and much nonwork, leisure and inefficiencies.« (Laffer 1979, 45) Insbesondere hätten die sich verringernden Nettoerträge auf die Produktionsfaktoren Arbeit und Kapiral zu verringertem Output, Beschäfigung und Einkommen geführt. Nicht nur wird die zunehmende Steuerbelastung produktiver Aktivitäten attackiert, sondern auch die Ausgabenseite des Staates inșbesondere die Transferzahlungen (im wesentlichen die Welfare-Ausgaben). Da Transferzahlungen durch Steuereinnahmen gedeckt werden müßten, seien Transferzahlungen »real resource transfers from producers and workers to recipients based upon some characteristics other than work or production ... That is transfers are often a payment explicitly for nonwork. Examples of this are agricultural subsidies, food stamps (income requirement) social security payments (retirement test) housing subsidies ... and obviously unemployment compensation« Laffer 1979, 47). In den Konzeptionen dieses weit verbreiteten Konservatismus in der ökonomischen Theorie erscheint nun nicht nur das »Nichtarbeiten《 als »selbst gewähî«, sondern auch als enorme Belastung der produktiven Aktivitäten.

- Ihr zweiter Angriff richtet sich gegen die antizyklische Nachfragepolitik des Staates. Antizyklische Ausgaben im Rahmen der Stabilisierungspolitik erscheinen in den Augen der neoklassischen Supply-Side-Ökonomen als »Inflationssteuer« (Mundel 1971, 33) für Besitzer produktiver Ressourcen. Überdies seien antizyklische Ausgaben auch ökonomisch unwirksam, denn die Ausgabenfinanzierung - über Steuererhöhungen würden Arbeits- und Investitionsanreize in der Krise vermindern und daher prozyklisch wirken, - über Difizitfinanzierung (Ausgabe von Staatsschuldpapieren) würde zum crowding-out von privaten Investitionen führen. Das Demand-Management ist in ihren Augen nicht nur unwirksam, sondern verstärkt die Rezession oder Krise.

- Die Wirtschaftspolitik muß nach ihrer Auffassung im wesentlichen an der AngeborsSeite ansetzen. Hierbei berufen sich die neuen Supply-Side-Ökonomen wieder auf das Say'sche Theorem, demzufolge die Produktion die Nachfrage schafft. (Vergessen wird hier wieder, daß dies nur bei einer geldlosen Wirtschaft zutreffen kann) Der Hauptpunkt ihres Wirtschaftsprogramms ist daher die Forderung nach radikaler Steuersenkung. Steuerentlastung für produktive Aktivitäten würde jedoch nicht über eine steigende Gesamtnachfrage im Keynesianischen Sinne Produktion, Beschäftigung und Einkommen erhöhen, sondern über einen erhöhten Anreiz zu produktiven Aktivitäten, da durch Steuersenkungen die Nettoerträge solcher Aktivitäten ansteigen würden. In ihren Augen sind es daher nicht die Nachfrageeffekte von Steuersenkungen, die wieder zur Vollbeschäftigung führen, sondern die Effekte auf der Produktionsseite. Auch die durch die Steuersenkungen verursachten staatlichen Defizite würden in langer Sicht wieder durch erhöhte Produktion, Beschäftigung, Einkommen und staatlichen Einnahmen abgebaut.

Wird diese übersimplifizierte Sicht ökonomischer Probleme des gegenwärtigen amerikanischen Kapitalismus sowohl von den Keynesianern (auch von den konservativen Keynesianern) und Gewerkschaften hefrig kritisiert, so hat die populistische Komponente in der Analyse der Supply-Side-Ökonomen ihnen nicht nur zu beträchtlicher Popularität und 
Unterstützung (insbesondere von Seiten der amerikanischen Mittelklassen) verholfen, sondern ihr Programm wird während der Präsidentschaftszeit Reagans auch überwiegend die praktische Wirtschaftspolitik bestimmen. Bevor ich auf die kommende praktische Wirtschaftspolitik der Reagan-Administration eingehe, will ich kurz die Charakteristika der Wirtschaftspolitik, Carters diskutieren.

\section{(III) Zur Praxis der Wirtschaftspolitik in den USA}

\section{Zur Wirtschaftspolitik der Carter-Administration}

Wenn Carter auch noch 1975 mit dem Programm der Erhöhung des Beschäftigungsniveaus seine Präsidentschaft angetreten hat, so hatten sich seine wirtschaftspolitischen Zielvorstellungen in den letzten Jahren aber sehr stark gewandelt. Seine Haushaltspolitik wurde mehr und mehr zu einer konservativen Austeritypolitik, die der Kritik der Monetaristen und konservativen Keynesianer entgegenkam. Der Primat der Stabilisierungspolitik wurde mehr und mehr die Inflationsbekämpfung. Die Wirtschaftspolitik selbst hatten aber nicht so sehr die Monetaristen oder die ultrakonservativen Vertreter der Rationalen-ErwartungsHypothese bestimmt oder gar die Supply-Side-Ökonomen, sondern - wie oben bereits erwähnt - die konservativen Keynesianer.

In wenigen Punkten möchte ich die Wirtschaftspolitik der Carter-Admimistration zusammenfassen (vgl. Council of Economic Advisors 1980):

Erstens: Einige Bemerkungen zur Geld- und Budgetpolitik: Carter benutzte durchaus noch die Instrumente der Stabilisierungspolitik. Insbesondere mittels sukzessiver Budgetkürzungen und einer harten Geldpolitik sollten die Inflationsraten gebremst und der Verfall des Dollars aufgehalten werden. Die Zeitabfolge der geldpolitischen Maßnabmen war jedoch mehr von außenwirtschaftlichen Erfordernissen (Verfall des Dollars) als durch das Ziel der Inflationsbekämpfung bestimmt, obwohl mit diesen Maßnahmen auch gleichzeitig interne Effekte angestrebt wurden. So z.B. wurden im Oktober 1979 der Diskontsatz auf $12 \%$ und die Mindestreservesätze auf $8 \%$ erhöht. Die Prime Rate ging im Dezember 1979 auf eine Rekordhöhe von 15 \% hoch, ebenso stiegen die kurzfristigen Zinsen an. Im März 1980 wurden noch schärfere Maßnahmen durchgeführt. Kreditkontrollen sollten die Konsumentenkredite verknappen, die Erhöhung der Mindestreservesätze auf $10 \%$ und die Erhöhung des Diskontsatzes auf 20 \% sollten das Geldangebot verknappen, die Zinssätze ansteigen lassen und ausländisches Geldkapital anlocken, dem Verfall des Dollars und der in diesem Jahr erwarteten 18-\%-Inflationsrate entgegenwirken. Die Prime Rate stieg daraufhin auf $20 \%$; dies ließ nicht nur die Industrieinvestitionen rapide fallen, sondern auch die gesamte Bauindustrie geriet in eine tiefe Krise. Die Prime Rate sank im Sommer 1980 zwar wieder ab, um dann aber aufgrund neuer geldpolitischer Maßnahmen im Dezember 1980 auf $21 \%$ anzusteigen.

Die Haushaltspolitik war schon seit 1977/78 von dem Versuch bestimmt, das durch die Krise 1975 entstandene große Budget-Defizit von über 70 Mrd Dollar durch Budgetkürzungen sukzessive wieder zu vermindern. Betrugen die Defizite 1975 noch 70 Mrd Dollar, 1976 noch über $60 \mathrm{Mrd}$, so fielen sie 1977 auf $37 \mathrm{Mrd}, 1978$ auf $26 \mathrm{Mrd}$, 1979 auf 27 Mrd und sind für 1980 bereits wieder auf über 40 Mrd prognostiziert. 
Wesentliche Bereiche der Kürzungen waren in den letzten zwei Jahren das Erziehungs- und Ausbildungsprogramm, das Gesundheitswesen (Medicaid und Medicare), Stadt- und Kommunalprogramme und Welfare-Ausgaben (Arbeitslosenunterstützung, Lebensmittelmarken, soziale Sicherheit). Sowohl im Carter-Programm für 1981, aber noch mehr im Haushaltsprogramm des Senats war bereits eine wesentliche Erhöhung des Militärhaushalts vorgesehen (Carter von 146,3 auf $150 \mathrm{Mrd}$, der Senat auf 155,7 Mrd Dollar).

Zweitens: Unter dem Einfluß der konservativen Keynesianer wurde aber gleichzeitig ein Programm zur Verbesserung der US-Konkurrenzposition auf dem Weltmarkt diskutiert. Man sprach von einer »Reindustrialisierung« der USA. Dieses Programm sollte die private Kapitalbildung stimulieren und das Wachstum der Produktivität fördern. So formuliert Carter etwa im Economic Report of the President: $\gg$ We must pursue measures to encourage productivity growth, adapt our economy rapidly to the fact of scarcer oil supplies, and improve our competitive standing in the world economy.« (Economic Report of the President 1980, 5). Zu diesem Zwecke sollten Forschung und Entwicklung gefördert und Steuererleichterungen für die Kapitalbildung vorgesehen werden (Investment Tax Credit; Einkommensteuersenkung, beschleunigte Abschreibung).

Drittens: Die konservativen Keynesianer, die vor der Einleitung einer drastischen Krise durch eine Stabilisierungspolitik warnten, weil die Kosten einer drastischen Rezession zu hoch seien, konnten sich in der Carter-Admimistration nicht voll durchsetzen. Von Carter und seinen ökonomischen Beratern sowie von einer konservativen Mehrheit des Kongresses und der Zentralbank (die letzten Jahres mit einem Vertreter einer harten Geldpolitik besetzt wurde, nämlich Volker) wurde ein Programm der Austerity bevorzugt, um durch eine Rezession die Preis- und Lohnbewegung zu stabilisieren. Der Alternative, favorisiert von den vielen Keynesianern, wurde nur teilweise gefolgt. Ein vierter Punkt wird in diesem Zusammenhang wichtig. Infolge des teilweisen Zerfalls des Konsensus zwischen amerikanischen Gewerkschaften und den großen Konzernen in den 70er Jahren - Folge der niedrigeren Wachstumsraten, der verschärften Auslandskonkurrenz und der geringeren Profitmargen - mußten korporativistische Strukturen geplant werden (wie z.B. der Pay-Advisory-Board, aber auch der Price-Advisory-Board), die den vollständigen Zerfall des Konsensus verhindern sollten. Diese korporativistischen Tendenzen (vgl. Economic Report of the President 1980) sind hier nun aber nicht mehr das Ergebnis progressiver keynesianischer Wirtschaftsplanung (wie in Europa während der Nachkriegszeit), sondern das Ergebnis einer Krisensituation und Austeritypolitik. Weitere korporativistische Strukturen (trilaterale Kommissionen für bedrohte Industriezweige) waren insbesondere mit den geplanten Reindustrialisierungsprogrammen vorgesehen.

Gegen dieses Programm der Carter-Administration, das die Krise der amerikanischen Wirtschaft im Jahre 1980 mit etwa 8 bis 8,5\% Arbeitslosen zwar nicht verursachte, aber zur Schärfe der Krise beitrug, war von seiten liberaler Keynesianer, von seiten des Kennedy-Flügels bei den Demokraten und vor allem von seiten der Gewerkschaften, Konsumentenorganisationen und der Intellektuellen Opposition erhoben worden. In vielen Punkten (z.B. in der Fiskalpolitik und in dem Versuch der Einschränkung der Gewerkschaftsmacht) hatte die Carter-Administration bereits eine Wirtschaftspolitik betrieben, die von dem wirtschaftspolitischen Programm Reagans nicht seht verschieden war. (So wundert es auch 
nicht, daß Reagan in seiner Wahlkampagne die traditionelle Basis der demokratischen Partei-Gewerkschaften und Minoritätsgruppen - entweder neutralisieren oder spalten konnte und die Präsidentschaftswahl schließlich gewann.)

\section{Zur Wirtschaftspolitik der Reagan-Administration}

Mit dem Wahlsieg Reagans wurde der konservative Trend in der amerikanischen Wirtschaftspolitik noch verstärkt. Konservative und neokonservative Vertreter der amerikanischen ökonomischen Theorie - die Supply-Side-Ökonomen - werden nun als Berater von Reagan im wesentlichen die zukünftige Wirtschaftspolitik der USA bestimmen. Obwohl im Reagan-Lager sowohl während der Präsidentschaftskampagne als auch während der Zeit der neuen Kabinettsbildung noch ein gewisser Widerspruch zwischen den alten gemäßigten Konservativen (wie A. Greenspan und G.P. Shultz) und den radikalen Neokonservativen (wie Ture, Laffer, Roberts, Mundel) sichtbar war, so haben letztendlich doch die jungen neoklassisch orientierten Supply-Side Ökonomen überwiegend das Reagan-Wirtschaftsprogramm bestimmt. Ihr Hauptprogrammpunkt ist die Beendigung der diskretionären Stabilisierungspolitik und eine langfristig orientierte Reduktion der "Belastungen" der Angebotsseite. Sie befürworten wie die Monetaristen und die Vertreter der Rationalen-Erwartungs-Hypothese eine harte Geldpolitik, um die zweistelligen Inflationsraten zu reduzieren, darüberhinaus aber auch drastische Steuersenkungen und Haushaltskürzungen, um über höhere Nettoerträge auf die Produktionsfaktoren speziell die Angebotsseite zu stimulieren.

Im Einzelnen sind folgende Maßnahmen geplant:

- Kernpunkt des Programms der Supply-Side Ökonomen ist eine drastische Steuersenkung, um die Arbeitsmotivation zu steigern und die Kapitalinvestitionen zu erhöhen. Republikanische Kongressabgeordnete haben auch bereits eine Gesetzesvorlage zur 30prozentigen Einkommenssteuersenkung (über die nächsten drei Jahre) im Kongreß eingebracht (Kemp / Roth-Tax Bill). Überdies sind eine beschleunigte Abschreibung, Investitions-, Forschungs- und Enrwicklungskredite sowie Steuersenkungen für Kapitalgewinne geplant. (Das von Reagan während der Wahlkampagne vertretene populistisch orientierte Programm der generellen Einkommenssteuersenkung wird vermutlich aufgrund des gegenwärtig starken Drucks der »Corporations« mehr und mehr zugunsten der steuerlichen Entlastung der Profite der Kapitalgesellschaften verschoben werden.)

- Von den Supply-Side Ökonomen wird zwar angenommen, daß langfristig infolge erneuter Produktion und Investitionen die Steuereinnahmen des Staates wieder ansteigen (und somit die Defizite wieder abgebaut wetden können), kurzfristig sollen aber Haushaltskürzungen vorgenommen werden, um rapide anwachsende Haushaltsdefizite mit entsprechenden Preissteigerungen zu verhindern. Haushaltskürzungen sind insbesondere im Bereich der Wohlfahrtsausgaben, der Transferzahlungen und der staatlichen Zuschüsse für Beschäftigungs- und Ausbildungsprogramme (CETA) geplant. (Gleichzeitig sollen aber zur Wiederherstellung der amerikanischen Vormachtstellung in der Weltpolitik die Militärausgaben gesteigert werden, so daß z.Z. noch niemand weiß, auf welche anderen Bereiche noch weitere Haushaltskürzungen zukommen werden.)

- Entsprechend der Theorie der »Free-Market-Economy« ist eine starke Entregulierung 
privater Industrien (z.B. im Energiebereich Wegfall der Windfall-Profit Tax für die Ölkonzerne) und der Abbau der Belastungen von privaten Unternehmen durch Umwelts-Sicherheits- und Gesundheitsauflagen beabsichtigt. Ebenso ist aber auch der Wegfall der unter Carter eingeführten Lohn-Preis-Richtlinien geplant, um der FreeMarket-Economy« wieder mehr Raum zu geben.

- In bezug auf den Arbeitsmarkt sind weitere drastische Gesetzesänderungen vorgesehen. Insbesondere sollen - um die Konkurrenz unter den jugendlichen Beschäftigten und Nichtbeschäftigten und die Job-Chancen für Minderheiten zu steigern - Mindestlöhne für Jugendliche herabgesetzt und Transfer-Zahlungen eingeschränkt werden. Überdies soll - und dies trifft die Gewerkschaften besonders hart - der Davis / Bacon-Act, der eine Lohngarantie für gewerkschaftlich organisierte Arbeiter in der Bauindustrie implizierte, sukzessive abgeschafft werden.

- Mittels einer harten Geldpolitik soll die Wachstumsrate des Geldangebots sukzessive vermindert werden, um die zweistelligen Inflationsraten zu drücken und den internationalen Wert des Dollars zu stabilisieren. In der Diskussion ist unter den Supply-Side Ökonomen und den Reagan-Beratern überdies die Rückkehr zum Goldstandard im internationalen Währungssystem (vgl. Mundel).

Auf dieses radikale Supply-Side orientierte Programm - das in vielen Punkten dem Thatcher-Programm ähnelt, jedoch mehr populistische Komponenten (wie z.B. die generelle Einkommenssteuersenkung) aufweist - haben die Keynesianer bisher nur sehr verhalten reagiert. Ihre Hauptkritikpunkte sind: Die expansiven Effekte eines Supply-Side orientierten Programms werden nicht sehr bedeutsam sein, die Inflationsrate wird durch die Steuersenkung und den Wegfall der Lohn-Preis-Richtlinien weiter ansteigen und die Haushaltsdefizite werden zu - statt abnehmen. Auf seiten einiger Reagan-Berater versucht man zwar bereits von dem englischen Mißerfolg zu lernen und zusätzlich zu dem langfristig orientierten neoklassischen Programm auch ein kurzfristig orientiertes Emergency - und Recovery Programm zu verabschieden, die Durchsetzungschancen eines solchen Programms sind aber als nicht sehr realistisch einzuschätzen. Von vielen Kritikern wird daher bereits prognostiziert, daß mit der Rücknahme von Staatsinterventionen, staatlichen Regulierungen und det keynesianischen Stabilisierungspolitik sich die sozialen Gegensätze unter Reagan soweit verschärfen werden, daß die USA in den 80er Jahren einen neuen Ausbruch von Revolten von Minderheitsgruppen erleben werden.

\section{(IV) Alternativvorstellungen der amerikanischen Gewerkschafien}

Da es im Gegensatz zur BRD in den USA nicht eine breit angelegte und organisierte Diskussion zu Alternativen der Wirtschaftspolitik gibt und auch keine ausformulierten Alternativprogramme existieren, will ich in diesem letzten Teil in einigen Punkten Alternativvorstellungen von den amerikanischen Gewerkschaften und von der außerinstitutionellen amerikanischen Linken, die seit dem konservativen Schwenk in der Carter-Wirtschaftspolitik diskutiert wurden, zusammenfassen.

Waren die Gewerkschaften in den frühen 70er Jahren noch ausschließlich an dem keynesianischen Nachfragemanagement als Mittel der Erhaltung der Beschäftigung orientiert, so machten sie in den 70er Jahren Lemprozesse durch, die eine leichte Verschiebung ihres Programms beobachten lassen. Auch sie sahen, wie die konservativen Keynesianer, die Defizite der amerikanischen Ökonomie nicht länger allein in der Nachfrageseite, sondern 
auch in der Produktions- und Angebotsseite. Für sie waren es aber im wesentlichen die hochkonzentrierten, multinationalen amerikanischen Konzerne, die das Problem auf der Produktionsseite verursacht haben. Die Antworten, die die Gewerkschaften (oder im wesentlichen der AFL/CIO) auf die Probleme der amerikanischen Ökonomie Ende der 70er Jahre, die Wirtschaftspolitik der Carter-Regierung und der Supply-Side-Ökonomen gaben, waren aber überwiegend noch keynesianisch und kurzfristig orientiert (vgl Statement of Dr. R. Oswald March 25, 1980 and May 6, 1980).

Heftige Opposition - vor allem im Kongreß - erhoben sic bereits gegen das Carter-Programm der Budget-Kürzungen, der Hochzinspolitik und der Bevorzugung der privaten Unternehmen bei Steuererleichterungen (Investment Tax Credit). Statt die Inflation mit dem Mittel der schweren wirtschaftlichen Rezession zu bekämpfen - eingeleitet durch eine Austerity-Politik insbesondere im Bereich der sozialen Wohlfahrt und der sozialen Dienstleistungen -, traten die Gewerkschaften bereits unter Carter für ein Programm des expansiven Haushalts und der Niedrigzinspolitik ein, das die Arbeitslosigkeit vermindern und die Produktivität steigern sollte. Insbesondere mit Hilfe der Fiskalpolitik sollten

- erstens die Beschäftigung im öffentlichen Sektor ausgedehnt, der Wohnungsbau gefördert, spezielle Ausbildungs- und Arbeitsbeschaffungsprogramme für Minoritäten geschaffen, die Arbeitslosenunterstützung ausgeweitet und die finanzschwachen und von schweren Krisen betroffenen Städte und Kommunen subventioniert werden.

- zweitens die Investitionen in solche Bereiche gelenkt werden, wo in den 70er Jahren besondere Defizite zu verzeichnen waren (Dienstleistungsbereich, Energie, Wohnungsbau, Lebensmittelproduktion, öffentliches Transportsystem, Gesundheitsleistungen).

Ihre Forderung gerade nach einer expansiven Fiskalpolitik in Zeiten ansteigender Inflationsraten begründeten die Gewerkschaften mit dem Argument, daß die Inflationsraten in den 70er Jahren weder mit Lohnsteigerungen noch mit Haushaltsdefiziten begründbar seien. Überdies könnten nur produktive Investitionen die Inflationsrate niedrig halten und die internationale Konkurrenzpositionen verbessern helfen.

Zur Verhinderung der mit einem solchen expansiven Programm verbundenen Preissteigerungen sollten weiterhin Preiskontrollen - und wenn nötig auch Lohnkontrollen - eingeführt werden, die kurzfristig die Inflationsrate niedrig halten. Kontrollen der Preissteigerungen sollten speziell eingeführt werden, in solchen Kernbereichen, die in den letzten Jahren überwiegend zum Anstieg der Lebenshaltungskosten geführt haben, also für Lebensmittelpreise, Energiepreise, für Häuserbau und Wohnraum und für den Bereich der Gesundheitsleistungen, da hier die höchsten Preissteigerungen stattgefunden hatten. Um jedoch hier das Absinken der Angebotserhöhung zu verhiridern, sollten produktive Investitionen auf diesen Gebieten vom Staat gefördert werden. Auf der anderen Seite schloß dieses Programm der Inflationskontrolle nicht nur die Kontrolle von Preisen, sondern auch die Kontrolle von Kapitaleinkommen ein (Dividenden, Profite, Zinsen und andere Formen von Kapitaleinkommen, wie übermäßige Managergehälter etc.).

Gemäß der Analyse der amerikanischen Gewerkschaften hatten im wesentlichen überdies die hochkonzentrierten, diversifizierten und international mobilen amerikanischen Konzerne die Probleme auf der Produktionsseite für die amerikanische Ökonomie geschaffen. Amerikanische Konzerne tätigten seit Ende der 50er Jahre produktive Investitionen überwiegend in europäischen Konkurrenzländern oder in Niedriglohnländern. Die Wachstumsrate der Produktivität der amerikanischen Tochterfilialen der multinationalen Kon- 
zerne sank (vgl. Stahl- und Autoindustrie). Aufgrund dieser Tatsache forderten die amerikanischen Gewerkschaften daher vom Staat in den letzten Jahren zunehmend einen Protektionismus für amerikanische Industrien und Arbeiter. Es sollten:

- die internationalen Investitionen der amerikanischen Konzerne beschränkt und kon. trolliert,

- Importrestriktionen für bestimmte Industriezweige verstärkt (Einführung von Quoten, z.B. im Autoimport) und

- ausländische Konzerne gezwungen werden, statt in die USA zu exportieren, dort Industriebetriebe aufzubauen.

Auf dem AFL/CIO-Kongreß im letzten Jahr wurde z.B. folgende Resolution verabschiedet:

»During the 1970s the US lost its preeminent position in foreign trade. Huge trade deficits depreceated the value of the dollar. Foreign imports eroded American jobs as American firms exported technology, capital and production potential... The Congress and the Administration shouid pursue an international economic policy that will stop the destruction and the export of American jobs and the undermining of the nation's industrial base. This can be achieved by regulating the export of American technology and capital, eliminating tax and other incentives that encourage US companies to establish and expand their operations in foreign countries and regulating the flood of imported goods and components that displace US productions... Imports, exports, technology transfer, money flows and investment must be reported in more detail, monitored and regulatet...\& (AFL/CIO, 1979, 1)

Wie aus diesem Zitat ersichtlich wird, ging es den Gewerkschaften also nicht nur um Importkontrollen, sondern um die Kontrolle der Kapitalmobilität. Allerdings wurden hier nur staatliche Kontrollen verlangt. Die Forderung nach Kontrolle der Kapitalmobilität innerhalb der großen Kapitalgesellschaften war eine der Forderungen, die zunehmend von anderen Gruppierungen außerhalb des AFL/CIO - die aber mit Gewerkschaftsverbänden zusammenarbeiten (vgl. Progressive Alliance und Bluestone / Harrison 1980) - aufgestellt wurde. Insbeondere gegen die seit Beginn der 70er Jahre ansteigenden Betriebsstillegungen (speziell im industrialisierten Norden und Nordwesten der USA) von Filialen der Großkonzerne wurde die Forderung nach Kontrolle der Kapitalmobilität entwickelt, (Bluestone / Harrison 1980). Langfristig gesehen, ist dies sicherlich eine richtige Forderung von seiten der Gewerkschaften, während der hereingebrochenen Krise 1980 war sie allerdings nur wenig realistisch, da aufgrund der Krise Betriebsstillegungen mit Massenentlassungen von 10-20000 (etwa in der Auto- und Stahlindustrie) Arbeitern stattfanden.

Neben diesen Kernpunkten gewerkschaftlicher Alternativprogramme gegen Krise und Arbeitslosigkeit waren natürlich noch andere - zukünftig auch sicherlich sehr relevante - Forderungen sowohl der Gewerkschaften als auch außerinstitutioneller Linker, Konsumentenund Community-Bewegungen und politischer Gruppierungen in der Diskussion. Der $\mathrm{AFL} / \mathrm{CIO}$ und noch mehr starke progressive Einzelgewerkschaften (UAW und Machinists) diskutierten z.B. durchaus auch die Forderung nach Arbeitszeitverkürzung als Mittel der Erhöhung des Beschäftigungsniveaus (Kürzung der Wochenarbeitszeit, Herabsetzung des Rentenalters, Urlaubsverlängerung). Andere Forderungen waren: Aufbau eines Frühwarnsystems bei drohenden Betriebsstillegungen, Ausdehnung der gewerkschaftlichen Repräsentation im »Board« der Konzerne, Ausdehnung der gewerkschaftlichen Rechte im Unternehmen durch Kongreßgesetzgebung (Refcrm des Wagner-Acts von 1935) und Nationalisierung der Ölkonzerne. Aber für solche Forderungen bestand infolge der Zersplitterung der amerikanischen Gewerkschaftsbewegung inerhalb des AFL/CIO oder in den Einzelge- 
werkschaften außerhalb des AFL/CIO entweder kein einheitlicher Konsens, oder die Gewerkschaften waren zu schwach, um diese Forderungen in der Öffentlichkeit populär zu machen oder Umsetzungsstrategien dafür zu entwickeln.

Zusammenfassend läßt sich sagen, daß die gewerkschaftlichen Alternativvorstellungen seit dem konservativen Schwenk in der amerikanischen Wirtschaftspolitik weitgehend kurzfri. stig orientiert waren. Wo jedoch von seiten der Gewerkschaften das Problem der Produktions- und Angebotsseite thematisiert und analysiert wurde (nämlich »was« und »wie« produziert wird), wurden keine Strategien diskutiert, die die gewerkschaftliche Kontrolle über die Produktions- und Angebotsseite hätten ausdehnen können. Dies sollte im wesentlichen dem betrieblichen Management überlassen werden.

Das mehr langfristige Problem der Ausdehnung der Kontrolle der Arbeiter und Gewerkschaften über die Produktions- und Angebotsseite durch »Economic Democracy« (Carnoy 1 Shearer 1980) und "Workplace Democracy« (Investitionsplanung, soziale Kontrolle der Investitionen, Ausdehnung der betrieblichen Partizipationsformen von Arbeitern, Arbeiterkontrolle und Arbeiter-Management-Komittees) sowie die Forderung nach Ausdehnung der öffentlichen Unternehmen, Aufbau von Modellbetrieben, die durch Arbeiter geleitet werden, Industrieansiedlungs- und Kommunalplanung durch Kommunen und Arbeiter (Institute für Economic Alternatives in Washington) und der Aufbau von Produzentenund Konsumenten-Kooperativen wird meist von Akademikern, kleinen politischen Gruppierungen und der außerinstitutionellen Linken thematisiert. Ihr Einfluß auf politisch bedeutsame Alternativprogramme in den USA ist gegenwärtig aber noch recht minimal, wenngleich auch bereits feste Verbindungen zu progressiven Einzelgewerkschaften (UAW und Machinists) bestehen (Progressive Alliance), die zukünftig sehr bedeutsam sein könnten. Insgesamt sind aber diese Alternativkonzeptionen sowohl der Gewerkschaften als auch linker Intellektueller nicht so sehr vom Bewußtsein und Standpunkt der Produzenten - wie z.B. mehr in der europäischen Diskussion zur alternativen Wirtschaftspolitik -, sondern mehr unter Anknüpfung an die traditionell seit der Weltwirtschaftskrise in den dreißiger Jahren sehr starke Konsumenten- und Community-Bewegung formuliert.

Eine andere Frage jedoch ist, inwieweit die seit Mitte der 70er Jahre wieder stärker aufkommenden alternativen Forderungen seitens der Gewerkschaften und Intellektueller eine Chance haben, zumindest partiell durchgesetzt werden zu können. Hierzu muß gesagt werden, daß die amerikanische Gewerkschaftsbewegung gegenwärtig so geschwächt ist, daß die Verwirklichungschancen solcher Alternativprogramme recht minimal sind. Die gegenwärtige Schwächung der amerikanischen Gewerkschaftsbewegung nicht nur durch die Spaltung und Zersplitterung der amerikanischen Arbeiterschaft in verschiedene ethnische Gruppen, und durch die gegenwärtige Krise und Massenarbeitslosigkeit, sondern auch durch die Ausweitung antigewerkschaftlicher legislativer Maßnahmen auf Bundes- und Einzelstaatsebene (z.B. Aushöhlung des Davis/Bacon Acts) erweist sich als sehr drastisch. Die Schwäche der amerikanischen Gewerkschaftsbewegung ist aber auch Folge systematischer Managementstrategien, Betriebe zu »Entgewerkschaftlichen«, sowie Folge des Aufkommens neuer, nichtorganisierter Industriezweige (Dienstleistungssektor). Die Mitgliederzahlen der amerikanischen Gewerkschaften fallen seit den 60er Jahren, so daß z.Zt. weniger als $20 \%$ der amerikanischen Arbeiter gewerkschaftlich organisiert sind. Hinzu kommt, daß die gegenwärtige Krise mit Massenentlassungen in der Auto- und Stahlindustrie die Mitgliederbasis der entsprechenden Gewerkschaften direkt drastisch hat zusammenschmelzen lassen. Eine starke Opposition war weder gegen die Carter-Wirtschaftspolitik 
der letzten Jahre sichtbar gewesen, noch wird von Kennern der amerikanischen Gewerkschaftsbewegung erwarter, daß Alternativen zum Reagan-Programm von Seiren der Gewerkschaften praktische Relevanz erhalten werden. Die amerikanische Gewerkschaftsbewegung isr gegenwärtig in einer Position, die es nicht erlaubt, auch nur ansatzweise Alrernativen der Wirtschafrspolitik durchzusetzen oder die neuen wirtschaftspolitischen Konzeptionen zur Reindustrialisierung der USA wesentlich zu beeinflussen. Auch wird nicht erwartet, daßs sie der neuen Supply-Side orientierten Wirtschaftspolitik Reagans großen Widerstand entgegensetzen werden können.

\section{Anmerkungen}

1 Für hilfreiche Diskussionen zu früheren Fassungen des vorliegenden Artikels möchte ich vor allem A.Shaikh, D.Gordon, J.Rogers, J.Hoffmann, E.Altvater und anderen Kollegen von der New School für Social Research in New York und der Freien Universität Berlin danken. Eine Langfassung des hier abgedruckten Artikels mit ausführlichen ökonomischen Daten ist erhältlich vom Autor.

2 E.ine andere Position vertritt z.B. D.Gordon (1980), der davon ausgeht, daß der Anstieg des nichtproduktiven, mit Überwachungstätigkeiten beschäftigten Personals in den großen Firmen, den Abfall der Arbeitsproduktivität in den 70er Jahren verursacht habe.

3 Zur abfallenden Konkurrenzfähigkeit der US-Ökonomie und ihrer internen politischen Folgen, vg1. Markovits / Ertman 1980

\section{Literatur}

AFL/CIO 1979, Resolution adopted by the Thirteenth Constitutional Convention of the AFL/CIO, Washington D.C. Noyember 1979

Baily 1978, M.N. Baily, Stabilization Policy and Private Economic Bebavior, in: Brooking Papers on Econimic Activity No. 1

Barro 1974, R.J. Barro, Are Government Bonds Net Wealth? in: Journa! of Political Economy November/December 1974

Barro /Grossman 1971, R.J. Barro / H.J.Grossmann, A General Disequilibrium Model of Income and Employment, in: American Economic Review, March 1971, 61 (1)

Benassy 1975, J.-P.Benassy, Neo-Keynesian Disequilibrium Theory in Monetary Economy, in: Review of Economic Studies October 1975, 42 (4)

Blinder / Solow 1974, A.S.Blinder / R.M.Solow, Analytical Foundations of Fiscal Policy, in: Brooking Institution, The Economics of Public Finance Washington 
Blinder / Solow 1973, Does Fiscal Policy Matter? in: Journal of Public Economics 2

Bluestone / Harrison 1980, B.Bluestone / B.Harrison Capital and Community, The Causes and Consequences of Private Disinventment, A Progressive Alliance Publication, Washingon D.C.

Boddy / Crotty 1975, R.Boddy / J. Crotty Class Conflict and Macropolicy: The Political Business Cyc$l e$, in: RURPE Vol 7 No. 1 (Spring 1975)

Bosworth 1980, B.P.Bosworth Economic Policy, in: J.A. Pechman (ed.), Setting National Priorities, Agenda for the 1980s. The Brooking Institution

Carnoy / Shearer 1980, M.Carnoy / D.Shearer, Economic Democracy. The Cballenge of the 1980s (M.L.Sharpe) New York

Clowet 1965, R.W.Clower, The Keynesian Counterrevolution: A Theoretical Appraisal, in: F.H.Hahn / F.R.R.Brechling (eds.) The Theory of Interest Rates London

Council of Economic Advisors 1980, Econimic Report of the President January 1980, Washington

Eichner 1976, A.S.Eichner, The Megacorp and Oligopoly Microfoundations of Macroeconomics, Cambridge / England

Feldstein 1973, M.Feldstein, Tax Incentives, Corporate Savings and Capital Accumulation in the United States, in: Journal of Public Economics April 1973

Feldstein / Summers 1977, M.Feldstein / L.Summers, Is the Rate of Profit Falling? Brooking Paper on Economic Activities No. 1

Fisher 1977, St.Fisher, Long-Term Contracts, Rational Expectations, and the Optimal Money Supply Rule, Vol. 85 , No. 1

Friedman,B, 1978, B.Friedman, Crowding Out or Crowding In. The Economic Corisequences of Financing Government Deficits, Discussion Paper No. 655, Harvard Institute of Economic Reseatch, Cambridge/USA

Friedman,M. 1968, M.Friedman, The Role of Monetary Policy, in: The American Economic Review March 1968, 58

Gordon 1981, Gordon,D. Capital-Labor, Conflict and the Productivity Slowdown, in: American Economic Review, Papers and Proceedings, May 1981

Grandmont / Laroque 1977, J.-M.Grandmont / G.Laroque, On Temporary Keynesian Equilibrium, in: G.C.Harcourt: The Microeconomic Foundations of Macroeconomics London 1977 (Macmillan)

Hatcourt 1977, G.C. Harcourt (ed.) The Microeconomic Foundations of Macroeconomics, London 1977

Hill 1979, T.P.Hill, Profits and Rates of Retum Organization for Economic Co-Operation and Development; Paris

Houthakker 1979, H.S.Houthakker, Growth and Inflation Analysis by Industry in: Brooking Papers on Economic Activity, No. 1

Joint Economic Committee 1979, Mid-Year Review of the Economy: The Outlook for 1979, Washington

Jonas 1976, R.J.Jonas, Auswirkungen des weltwirtschaftlicben Strukturwandels auf die Arbeitsmarktentwicklung in der BRD, in: H.Heidemann (Hrsg.): Wirtschaftsstruktur und Beschäftigung, BonnBad Godesberg

Kamrany / Chereb, Kamrany, N.M. / Chereb, D.M., Productivity Performance of the United States, in: Kamrany, N.M. / Day, R.H. (eds) Economic Issues of the Eighties, Baltimore 1979 (John Hopkins)

Knight 1975, E. Knight, Economic Policy and Inflation in the United States, A Survey of Developments ftom the Enactment of the Employment Act of 1946 trough 1974. A Study prepared for the

use of the Joint Economic Committee, Congress of the Unites States, April 7, 1975

Laffer 1979, An Equilibrium Rational Macroeconomic. Framework, in Kamatany / Chereb ...

Leijonhufvud 1968, A.Leijonhufvud, On Keynesian Economics and the Economics of Keynes, Oxford

Lovel 1978, M.C.Lovel, The Profit Picture. Trends and Cycles, Brooking Papers on Economic Activity No. 3

Lucas 1973, R.E.Lucas Jr., Some International Evidence on Output-Inflation Trade-Offs, in: American Economic Review vol. 63 
Maddison 1964, A.Maddison, Economic Growth in the West, New York

Mayer 1973, Mayer,H., Die Technologische Lücke zwischen der Bundesrepublik Deutscbland und den Vereinigten Staten von Amerika, Tübingen

Malinvaud 1977, E.Malinvaud, The Theory of Unemployment, Reconsidered, Oxford

Markovits / Ertman 1980, Markovits, A. / Ertman,Th., Das »Modell Deutschland", Eine Herausforderung für die U.S.A., in: Prokla Heft 41

Modigliani 1977, F.Modigliani, The Monetarist Controversy or, Should We Forsake Stabilization Poli. cies?, in: American Economic Review

Mundel 1971, Mundel,R.A., Monetary Theory, Inflation, Interest and Growth in the World Economy, Pacific Palisade (Good year)

Muth 1961, J.F.Muth, Rational Expectations and the Theory of Price Movements, in: Econometrica Vol. 29

Nordhaus 1974, W.O. Nordhaus, The Falling Share of Profits, Brooking Papers on Economic Activity, No. 1

Okun / Perry 1978, Okun,A. / Perry,L. (eds.), Inflation. Brooking Institute, Washington

Phelps 1970, E.S.Phelps (ed.), Microeconomic Foundations of Unemployment and Inflation Theory, New York

Pool 1976, W.Pool, Rational Expectations in the Macro Model, in: Brooking Papers on Economic Activity No. 2

Roberts 1978, Roberts,C.P., The Break down of the Keynesian Model, in: Public Interest

Sachs 1979, J.D.Sachs, Wages, Profits and Macroeconomic Adjustment. A Comparative Study in: Brooking Papers on Economic Activity No. 2

Sargent 1976, Th.J.Sargent, A Classical Macroeconometric Model for the United States, in: Journal of Political Economy Vol. 84, No. 21

Sargent / Wallace 1976, Th.J.Sargent / N.Wallace, Rational Expectations and the Theory of Economic Policy, in: Journal of Monetary Economics (2)

Seltzer 1978, R.Seltzer, The Development of Crisis in the United States, in: Economic Education Project of the URPE: U.S. Capitalism in Crisis New York

Semmler 1979, W.Semmler, Competition and Monopoly Power. Theories and Empirical Evidence A Reconsideration of the Center of Gravity Concept of the Classical and Marxian Political Economy Washingon, (im Erscheinen)

Shaikh 1979, A.Shaikh, Profitrate in the U.S., (unveröff. Manuskript), New York

Solow 1980, R.M.Solow, On Theories of Unemployment, in: The American Economics Review March 1980

Sylos-Labini 1969, P.Sylos-Labini, Oligopoly and Technical Progress, Cambridge/Mass.

Statement of Dr.A.Oswald (AFL/CIO) 1980, Statement of Dr.A. Oswald (AFL/CIO) to the Joint Economic Committee on Inflation, (Research Office of the AFL/CIO), March 25, 1980

Statement of Dr.A.Oswald (AFL/CIO) 1980/2, Statement of Dr.A. Oswald (AFL/CIO) on the Nationat Economy, (Research Office of the AFL/CIO), May 6, 1980

Statistical Abstracts 1979, U.S. Department of Commerce, Bureau of Census, Statistical Abstracts of the United States, Washington, D.C.

Statistisches Jahrbuch 1976, Statistisches Jabrbuch 1976, Statistisches Bundesamt, Wirtschaftskunde für die BRD

Tobin 1978, J.Tobin, Asset Accumulation and Economic Activity. Reflections on Contemporary Macroeconomic Theory, Yryö Jahnsson Lectures 1978, (unveröff. Manuskript)

Weintraub 1979, E.R.Weintraub, Microfoundations. The Compatibility of Microeconomics and Macroeconomics, Cambridge/England

Weisskopf 1979, Th.E.Weisskopf, Marxian Crisis Theory and the Rate of Profit in the Post-War U.S. Economy in: Cambridge Journal of Economics No. 3

Wanniski 1978, Wanniski,j., The Way the World Works, New York (Basic Books) 